\title{
Design, fabrication, and characterization of form-birefringent multilayer polarizing beam splitter
}

\author{
Rong-Chung Tyan \\ Department of Electrical and Computer Engineering, University of California at San Diego, La Jolla, \\ California 92093
}

Atul A. Salvekar, Hou-Pu Chou, Chuan-Cheng Cheng, and Axel Scherer

Department of Electrical Engineering, California Institute of Technology, Pasadena, California 91125

Pang-Chen Sun, Fang Xu, and Yeshayahu Fainman

Department of Electrical and Computer Engineering, University of California at San Diego, La Jolla, California 92093

Received September 13, 1996; accepted November 21, 1996

\begin{abstract}
Polarizing beam splitters that use the anisotropic spectral reflectivity (ASR) characteristic of high-spatialfrequency multilayer binary gratings have been designed, fabricated, and characterized. Using the ASR effect with rigorous coupled-wave analysis, we design an optical element that is transparent for TM polarization and reflective for TE polarization at an arbitrary incidence angle and operational wavelength. The experiments with the fabricated element demonstrate a high efficiency $(>97 \%)$, with polarization extinction ratios higher than 220:1 at a wavelength of $1.523 \mu \mathrm{m}$ over a $20^{\circ}$ angular bandwidth by means of the ASR characteristics of the device. These ASR devices combine many useful characteristics, such as compactness, low insertion loss, high efficiency, and broad angular and spectral bandwidth operations. (C) 1997 Optical Society of America [S0740-3232(97)01107-1]
\end{abstract}

\section{INTRODUCTION}

Numerous optical information processing and imaging systems employ different polarization states to increase the information bandwidth and to reduce the cross talk between different channels. Some application examples include free-space optical switching networks, ${ }^{1-3}$ readwrite magneto-optical data storage systems, ${ }^{4,5}$ and polarization-based imaging systems. ${ }^{6,7}$ In these systems, a polarizing beam splitter (PBS) is an essential element for separating two orthogonally polarized light beams. Most of the applications require that the PBS providing high extinction ratios tolerate a wide angular bandwidth for high-resolution imaging, a broad wavelength range for operation with broadband sources, and a compact size for effective packaging. Conventional PBS based on either natural crystal birefringence (e.g., Wollaston prisms) or polarization selectivity of multilayer structures (e.g., PBS cubes) do not meet these requirements. The Wollaston prism requires a large thickness to generate enough walk-off distance between the two orthogonal polarizations owing to the intrinsically small birefringence of naturally anisotropic materials. An alternative design of a Wollaston-type prism takes advantage of formbirefringent materials that possess birefringence several times larger than that of natural birefringent materials, reducing the thickness considerably. ${ }^{8}$ However, the fabrication of such a structure is a tedious and complicated process, since thousands of layers of thin-film slab need to be fabricated. PBS cubes are easier to fabricate, but they provide good extinction ratios only in a narrow angular bandwidth for a limited spectral range. ${ }^{9}$ Other designs that utilize form-birefringent high-spatial-frequency surface-relief gratings ${ }^{10-12}$ and a single-layer-coated dielectric slab ${ }^{13}$ have been proposed to reduce the size of the components, to solve the material compatibility problem, and to simplify the fabrication process. However, they usually suffer from low efficiency, low extinction ratio, small angular bandwidth, and operation in a limited wavelength range.

Previously ${ }^{14}$ we introduced a new PBS device that uses the unique properties of anisotropic spectral reflectivity (ASR) characteristics of a high-spatial-frequency multilayer binary grating. The ASR mechanism is based on combining the effects of the form birefringence of a high-spatial-frequency grating (i.e., grating period is much smaller than the wavelength of the incident field) with the resonant reflectivity of a multilayer structure. With our approach, the angular field and the wavelength range have been largely increased compared with conventional PBS devices. Many packaging and material compatibility problems have also been resolved with this new design. Some interesting characteristics of the element with ASR characteristics cannot be found in a conventional PBS component. For instance, when our ASR de- 
vice is designed to operate with normally incident light, it acts as a highly efficient polarization-selective mirror.

In this paper we report detailed design, fabrication, and experimental characterization of such a PBS based on ASR properties. In Section 2 we describe the principle of the ASR effect of a high-spatial-frequency multilayer binary grating. We employ the effective-medium theory $(\mathrm{EMT})^{15}$ to explain intuitively why high-spatial-frequency multilayer binary gratings possess such characteristics. In Section 3 we introduce the design methodology of the PBS that employs such ASR properties. Here rigorous coupled-wave analysis (RCWA) ${ }^{16}$ tools are used to optimize $^{17}$ the design of the PBS. We also investigate the angular and the wavelength dependence of the ASR PBS. In Section 4 the effects of various fabrication errors on performances of the PBS are studied. In Section 5 we discuss the fabrication techniques employed to make our PBS, and we present experimental characterization results. We evaluate our PBS design in terms of polarization extinction ratio and efficiency for operation with waves of wide angular bandwidth. We also compare the experimental results with the numerical predictions. The summary and the directions for future research are provided in Section 6.

\section{PRINCIPLES OF ANISOTROPIC SPECTRAL REFLECTIVITY}

For reviewing properties of wave propagation in stratified media, consider a multilayer structure formed on a sub-
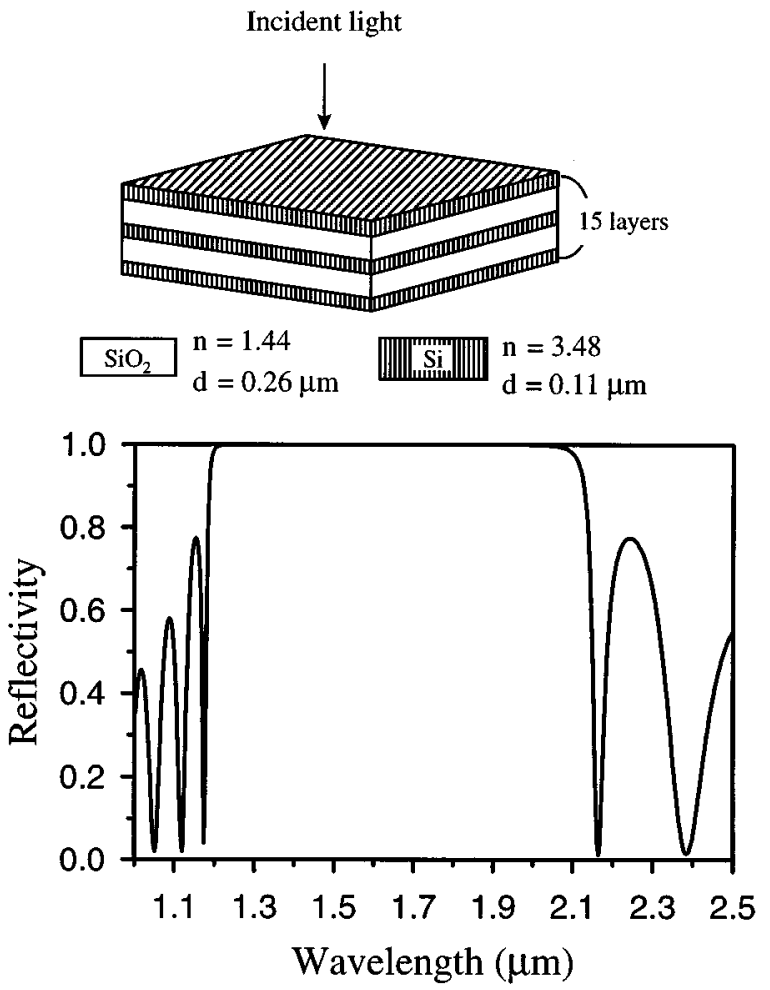

(a) strate by deposition of alternating layers of isotropic dielectric materials with high and low indices of refraction $n_{h}$ and $n_{l}$, respectively. Such a structure exhibits high reflectivity in a wide spectral bandwidth. When the thickness of each layer corresponds to a quarter-wave optical thickness for a selected wavelength, a highreflectivity spectral band will roughly center at that wavelength. For example, Fig. 1(a) shows a 15-layer quarter-wave structure made of $\mathrm{Si}$ and $\mathrm{SiO}_{2}$ with refractive indices of 3.48 and $1.44,{ }^{18}$ respectively, for an incident wavelength of $1.523 \mu \mathrm{m}$. A high-reflectivity spectral band is clearly shown in Fig. 1(a) near that wavelength. One can increase the reflectivity of such a quarter-wave layered structure by increasing the value of the ratio $n_{h} / n_{l}$ and the total number of layers. ${ }^{19}$ Furthermore, larger values of the ratio $n_{h} / n_{l}$ also increase the spectral bandwidth of high reflectivity.

For an optical field at a normal angle of incidence, a multilayer structure that is made of isotropic dielectric materials presents identical reflectivity spectra for any two orthogonal linear polarizations. This occurs because of the symmetry of the structure for a normally incident wave. Therefore the structure cannot be used to separate normally incident fields by polarization. By substituting one of the isotropic materials that are used to form the multilayer structure with birefringent materials, we can create a new multilayer structure that will possess reflectivity spectral bands centered at different wavelengths for the two orthogonal polarizations at normal incidence. It is illustrated in Fig. 1(b) that once one of the
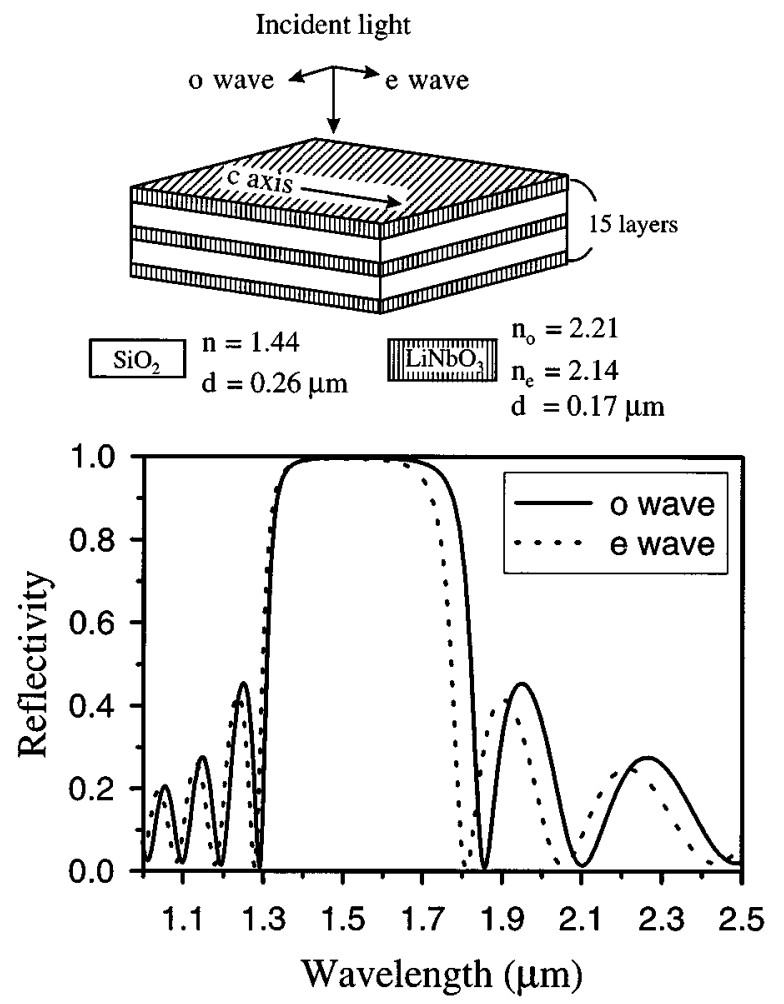

(b)

Fig. 1. (a) Schematic diagram of a 15-layer quarter-wave structure constructed of two isotropic materials $\left(\mathrm{Si}^{\mathrm{and}} \mathrm{SiO}\right)_{2}$; a plot of its spectral reflectivity is also shown. The thickness of $\mathrm{Si}$ and $\mathrm{SiO}_{2}$ layers are set as the quarter-wave optical thickness of the wavelength of $1.523 \mu \mathrm{m}$. (b) Same as (a), with $\mathrm{Si}$ being replaced by anisotropic material, $\mathrm{LiNbO}_{3}$. The thickness of the $\mathrm{LiNbO}_{3}$ layer is set as the quarter-wave optical thickness corresponding to the refractive index for the ordinary wave. 
isotropic materials (say, $\mathrm{Si}$ ) is replaced by an anisotropic material the spectral reflectivity bands are separated [in this example we disregard the material incompatibility problems and choose $\mathrm{LiNbO}_{3}$ with refractive indices of 2.21 and 2.14 (Ref. 20) for ordinary and extraordinary waves, respectively]. We call these phenomena ASR. Unfortunately, multilayer structures consisting of natural anisotropic materials cannot easily be fabricated. Furthermore, since natural anisotropic materials possess small birefringence, this separation will be small. With our approach based on a high-spatial-frequency multilayer binary grating, the separation between reflectivity spectral bands can increase considerably owing to the exceptionally large anisotropy ${ }^{21}$ that can be obtained with a form-birefringent nanostructure.

Form-birefringence effects ${ }^{22}$ appear in high-spatialfrequency gratings constructed of isotropic dielectric materials. Because of the geometric asymmetry of the grating structure, the two orthogonally polarized optical fields, one parallel to the grating grooves (called the TE field) and the other perpendicular to the grating grooves (called the TM field), encounter different boundary conditions, resulting in distinct effective indices of refraction. On propagation through the grating structure, the TE and the TM fields will acquire a relative phase difference similar to that obtained in natural anisotropic materials. This similarity takes place because the subwavelength structure can be designed such that only the zeroth diffraction order will propagate, while all the higher diffraction orders will become evanescent. Such a high-spatialfrequency grating at the boundary between two isotropic materials can be seen as an equivalent thin film of anisotropic material.

For normally incident TE- and TM-polarized optical waves, the effective indices of refraction of a surface-relief high-spatial-frequency binary grating can be estimated from the second-order $\mathrm{EMT}^{15}$ :

$$
\begin{aligned}
n_{\mathrm{TE}}^{(2)}= & {\left[n_{\mathrm{TE}}^{(0)^{2}}+\frac{1}{3}\left(\frac{\Lambda}{\lambda}\right)^{2} \pi^{2} F^{2}(1-F)^{2}\left(n_{\mathrm{III}}^{2}-n_{\mathrm{I}}^{2}\right)^{2}\right]^{1 / 2} } \\
n_{\mathrm{TM}}^{(2)}= & {\left[n_{\mathrm{TM}}^{(0)^{2}}+\frac{1}{3}\left(\frac{\Lambda}{\lambda}\right)^{2} \pi^{2} F^{2}(1-F)^{2}\left(\frac{1}{n_{\mathrm{III}}^{2}}-\frac{1}{n_{\mathrm{I}}^{2}}\right)^{2}\right.} \\
& \left.\times n_{\mathrm{TE}}^{(0)^{2}} n_{\mathrm{TM}}^{(0)^{6}}\right]^{1 / 2},
\end{aligned}
$$

where $F$ is the duty cycle of the grating defined by $F$ $=w / \Lambda$, with $w$ being the width of the binary grating [see Fig. 2(a)]; $\Lambda$ is the grating period; $\lambda$ is the wavelength of the incident wave; $n_{\mathrm{I}}$ and $n_{\mathrm{III}}$ are the indices of air and the grating material, respectively; and $n_{\mathrm{TE}}^{(0)}$ $=\left[F n_{\mathrm{III}}^{2}+(1-F) n_{\mathrm{I}}^{2}\right]^{1 / 2}$ and $n_{\mathrm{TM}}^{(0)}=\left\{n_{\mathrm{III}}^{2} n_{\mathrm{I}}^{2} /\left[F n_{\mathrm{I}}^{2}\right.\right.$ $\left.\left.+(1-F) n_{\mathrm{III}}^{2}\right]\right\}^{1 / 2}$ are the effective indices of refraction for TE and TM waves, respectively, provided by the zeroorder EMT. $^{22}$ When a high-spatial-frequency grating is formed in a multilayer structure made of two isotropic materials, the composition becomes an artificial anisotropic multilayer structure that will possess ASR characteristics. One can fabricate such an element by etching a high-spatial-frequency binary grating directly into a multilayer mirror structure.
Figure 2(a) shows an example of a high-spatialfrequency multilayer binary grating. The two isotropic materials used for constructing the multilayer structures are $\mathrm{SiO}_{2}$ and $\mathrm{Si}$, with refractive indices of 1.44 and 3.48, respectively, for an operating wavelength of $1.523 \mu \mathrm{m}$. The $\mathrm{SiO}_{2}$ and $\mathrm{Si}$ materials are chosen because of their fabrication compatibility and because of low absorption coefficients in the near-infrared region, which ensure a low insertion loss of the device. For operation as a formbirefringent zeroth-diffraction-order grating, we set the grating period to be equal to $0.6 \mu \mathrm{m}$, with the duty cycle of $F=0.5$. Using second-order EMT [Eqs. (1) and (2)], we

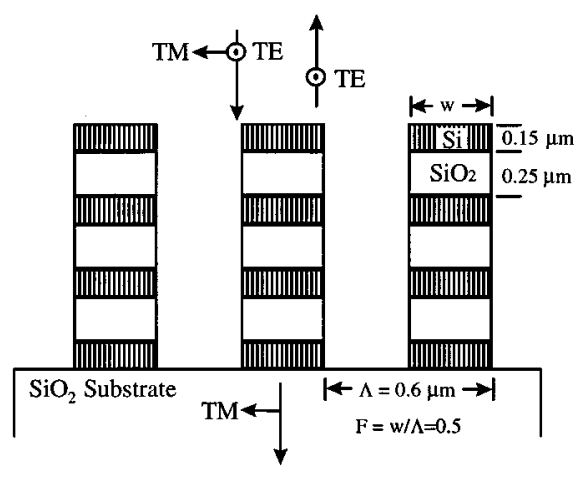

(a)

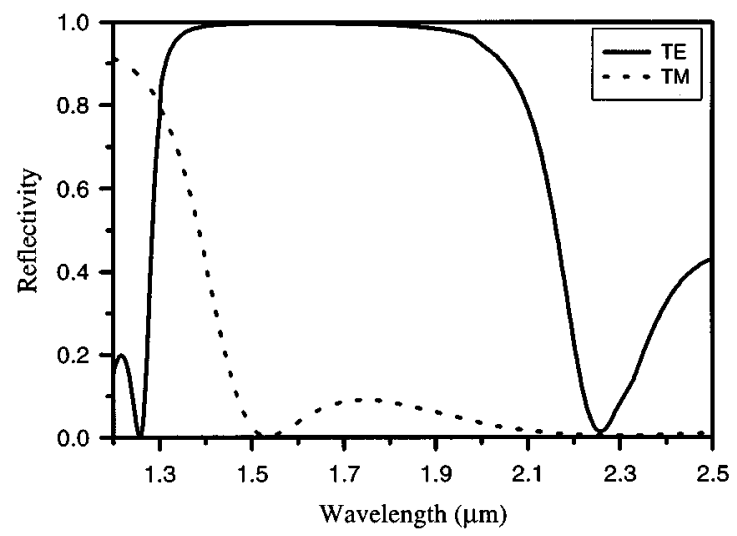

(b)

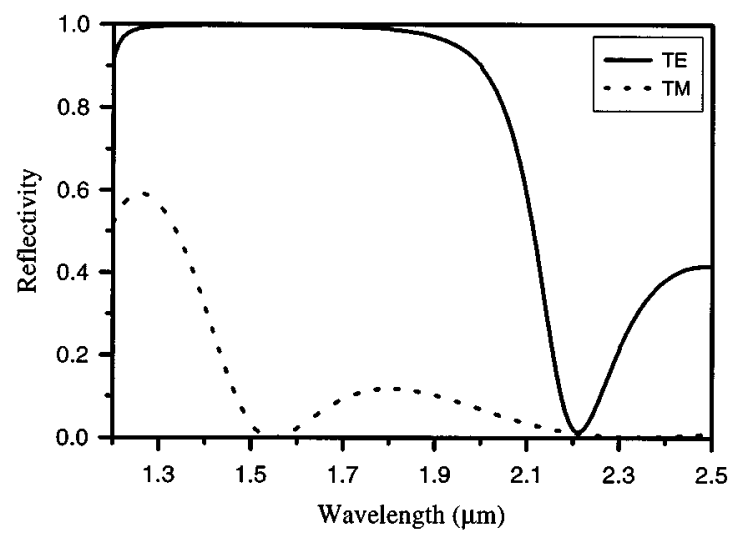

(c)

Fig. 2. (a) Schematic diagram of a seven-layer ASR PBS designed for light at normal incidence. The center operating wavelength is $1.523 \mu \mathrm{m}$. The design parameters are indicated in the figure. (b) EMT and (c) RCWA results of the reflectivity for TE- and TM-polarized waves versus wavelength. 
obtain the following effective refractive indices for the two materials: $\quad n_{\mathrm{TE}, \mathrm{Si}}^{(2)}=3.24, \quad n_{\mathrm{TE}, \mathrm{SiO}_{2}}^{(2)}=1.25$ and $n_{\mathrm{TM}, \mathrm{Si}}^{(2)}$ $=1.72, n_{\mathrm{TM}, \mathrm{SiO}_{2}}^{(2)}=1.18$. The effective indices of both materials used in the multilayer structure are larger for TE polarization than for TM polarization. This indicates that in the spectral domain the reflectivity band for TE polarization will be centered at a longer wavelength as compared with that for TM polarization, i.e., we will clearly observe a large ASR effect. The separation between the two reflectivity spectral bands has been dramatically increased over that possible with natural anisotropic materials.

The ASR characteristic of a multilayer formbirefringent binary grating is the essential property needed to realize the ASR PBS. By using the theory of optical wave propagation in a stratified medium ${ }^{23}$ that is characterized by second-order EMT, we show that the ASR property can be used to create a compact PBS. Figure 2(b) shows the spectral reflectivities of the sevenlayer grating illustrated in Fig. 2(a). It can clearly be seen that near a wavelength of $1.523 \mu \mathrm{m}$ the grating is transparent for TM polarization and reflective for TE polarization. Therefore the two orthogonal polarization components of the light wave can be separated to propagate in opposite directions with efficiencies close to $100 \%$.

\section{DESIGN AND MODELING OF THE POLARIZING BEAM SPLITTER}

In Section 2 we used second-order EMT for an intuitive explanation of the ASR characteristic of high-spatialfrequency gratings. However, previous studies ${ }^{17,24,25}$ have shown that, to accurately model devices in which the grating period is compatible with the wavelength, a rigorous method must be employed. In our case the grating period is approximately one third of a wavelength. Our modeling method is to use EMT results as an initial estimate of device parameters and then use a rigorous method to modify the design for optimal performance. The modeling tool that we used is RCWA, which we have experimentally verified for its accuracy in modeling of surface-relief-type grating. ${ }^{21,26}$

The design procedure is described by the following two steps: First, we use second-order EMT to calculate the effective indices of TE- and TM-polarized light in each layer of the grating at the desired center operating wavelength. The grating period is set to be small enough that only the zeroth diffraction order can propagate. From our previous study $^{17}$ we know that a high-spatialfrequency grating can possess the largest effective-index difference for waves with orthogonal polarizations when the duty cycle is near 0.5. Therefore we set the duty cycle $F=0.5$ as an initial value. An interesting characteristic is that the value of the effective-index ratios for TE-polarized light is larger than that for TM-polarized light. For example, effective-index ratios of the grating illustrated in Fig. 2(a), $\left(n_{h} / n_{l}\right)_{\mathrm{TE}}$ and $\left(n_{h} / n_{l}\right)_{\mathrm{TM}}$, are 2.59 and 1.46, respectively. This indicates that, to achieve the same reflectivity, the number of layers required for $\mathrm{TE}$ polarization will be less than that required for TM polarization. To minimize the number of layers needed to achieve a desired performance, we choose to maximize reflectivity for TE-polarized light.

In the next step we allow each layer to have a quarterwave optical thickness based on the effective index for TE-polarized light. These parameters are now used as the basis for an optimum design by RCWA. The thickness and the duty cycle of a high-spatial-frequency grating have been shown ${ }^{17}$ to be the most important parameters that will affect the phase difference between the two orthogonally polarized waves. Optimization is performed by incremental variation of the thickness of the layers of the grating to obtain the highest extinction ratio at the operational wavelength. To achieve broad reflectivity spectral bands, we use high-refractive-index materials for both the first and the last layers in the structure. ${ }^{19}$

Figure 2(c) shows the RCWA results of TE and TM reflectivities as a function of the wavelength for the sevenlayer ASR PBS shown in Fig. 2(a). The PBS is designed for normally incident optical fields at a center operating wavelength of $1.523 \mu \mathrm{m}$. As expected, the reflectivity of TE-polarized light is higher than that of TM-polarized light. The TE reflectivity spectral band is broader and is centered at a longer wavelength than is that of the TM wave. This ASR property cannot be accomplished with an isotropic multilayer structure for a normally incident optical field. Since this PBS is made of nonabsorbing materials (at those operating wavelengths), there is basically no insertion loss, and the efficiency is nearly $100 \%$. Looking at Fig. 2(c), we can see that both the TE reflection efficiency and the TM transmission efficiency are higher than $99 \%$ at the operating wavelength of 1.523 $\mu \mathrm{m}$. Also, the transmission polarization extinction ratios (defined as the ratio of transmittance of TM-polarized light to that of TE-polarized light) remain high over a broad spectral range ( $>200: 1$ over a $200-\mathrm{nm}$ range). However, the reflection polarization extinction ratios (defined as the ratio of reflectance of TE-polarized light to that of TM-polarized light) are extremely high over a small spectral range ( $>1000: 1$ over a $20-\mathrm{nm}$ range). These unique features can be employed in constructing either broadband low-insertion-loss normal-incidence polarizers or highly efficient polarization-selective mirrors for microlaser cavities. Other ASR PBS designs following the design procedure mentioned above for a center operating wavelength of $1.3 \mu \mathrm{m}$ can be found in our previous paper. ${ }^{14}$

To separate the path of the reflected wave from that of the incident wave, we also investigate an off-axis geometry [Fig. 3(a)]. Figure 3(b) shows the numerical results of the reflectivity versus the wavelength of the slanted incidence optical wave from a five-layer grating. For an incident wavelength of $1.523 \mu \mathrm{m}$, both the TE reflection efficiency and the TM transmission efficiency are higher than $99 \%$, and the polarization extinction ratios for reflection and transmission are better than 800:1 and 300:1, respectively. This slanted incidence arrangement offers two advantages: (1) Reflectivity from each layer for TE polarization is increased; thus only five layers were needed to achieve performance similar to those of the seven-layer design for normally incident light; and (2) the sidelobe for the TM reflectivity is flattened, allowing op- 


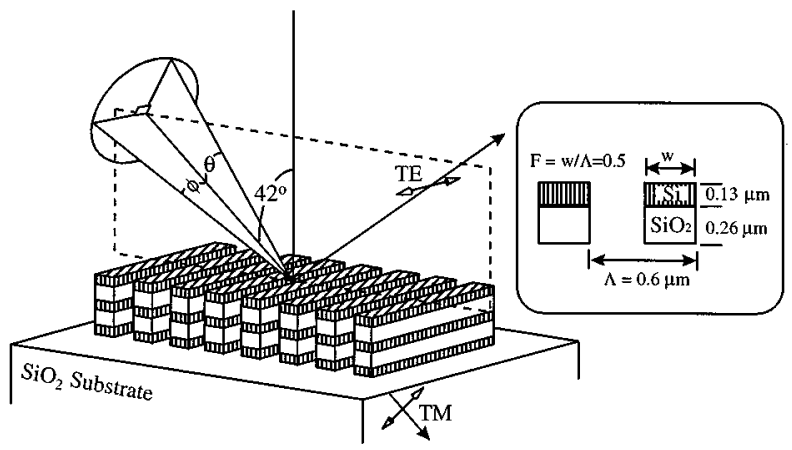

(a)

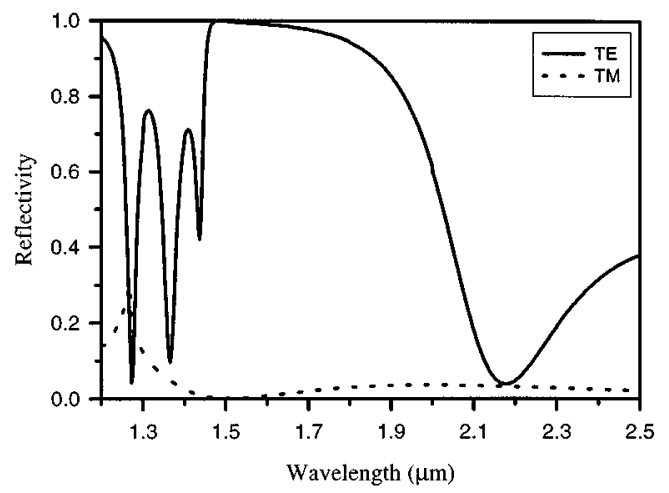

(b)
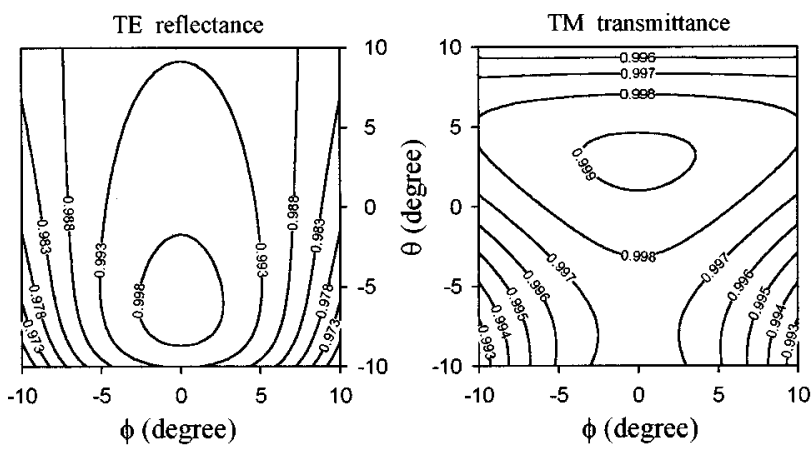

(c)
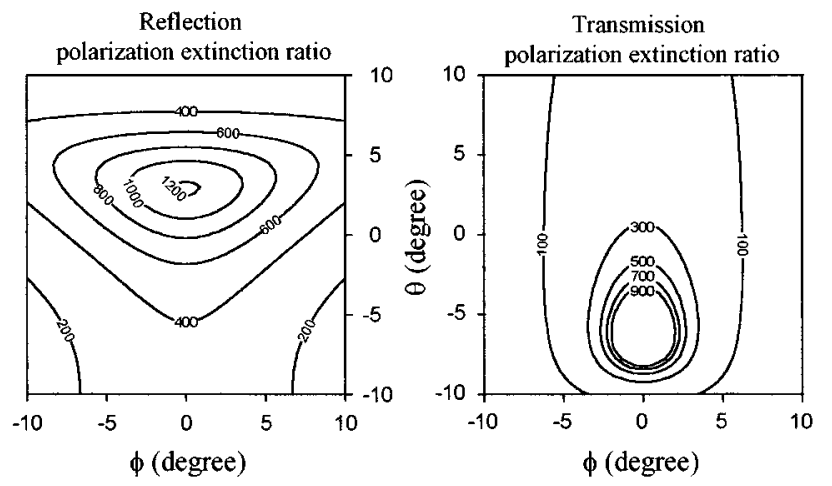

(d)

Fig. 3. (a) Schematic diagram of a five-layer ASR PBS operating with waves incident at an angle of $42^{\circ}$. The center operating wavelength is $1.523 \mu \mathrm{m}$. The design parameters are indicated in the figure. (b) RCWA results for the reflectivity of TE- and TM-polarized waves versus wavelength. The angles of $\theta$ and $\phi$ are zeros in this calculation. Contour plots of (c) TE reflectance and TM transmittance and (d) the reflection and transmission polarization extinction ratio versus angles $(\phi, \theta)$ are as defined in Fig. 3(a). The angles of incident waves are varied to span an angular bandwidth of $\pm 10^{\circ}$ in both $\theta$ and $\phi$ directions near the initial bias angle of $42^{\circ}$.

eration of the beam splitter in an even broader spectral range. One can further improve the efficiencies and the extinction ratios of the PBS by fine tuning the grating period and the duty cycle or by adding more layers to the structure.

Various applications require that the optical components in the system have abilities to maintain good performance within a large two-dimensional (2D) angular bandwidth and broad spectral range of light waves. Conventional PBS's provide good extinction ratios, usually in a narrow angular bandwidth for a limited wavelength range. Most of the polarization-sensitive diffractive optical elements also lack these abilities. Our PBS, however, is shown to provide good performances for optical signals that have a wide 2D angular bandwidth, as well as a broad spectral range. As illustrated in Fig. 3(a), the angles of incidence are varied to span an angular bandwidth of $\pm 10^{\circ}$ in both $\theta$ and $\phi$ directions defined near the initial $42^{\circ}$ bias angle. The results shown in Fig. 3(c) indicate that, at a wavelength of $1.523 \mu \mathrm{m}$, the TE reflection efficiencies are higher than $99.2 \%$ and $98 \%$ in the $5^{\circ}$ and $10^{\circ}$ angular bandwidth cones, respectively, and the TM transmission efficiencies are higher than $99.6 \%$ in both these angular ranges. The polarization extinction ratios [see Fig. 3(d)] are better than 400:1 and 200:1 for reflection in the $5^{\circ}$ and the $10^{\circ}$ angular bandwidth cones, respectively. For the transmission, the polarization ex- tinction ratios are smaller but still better than 130:1 and $50: 1$ in the $5^{\circ}$ and the $10^{\circ}$ angular bandwidth cones, respectively. These results show that a wide $2 \mathrm{D}$ angular bandwidth, as well as a broad spectral range, of operation is possible with this design. Furthermore, the property of high and uniform efficiency makes the ASR PBS suitable for many imaging systems applications.

\section{STUDIES OF FABRICATION TOLERANCES OF THE POLARIZING BEAM SPLITTER}

In this section we investigate the effect of fabrication errors (e.g., etch depth error, grating profile error, and duty cycle error) on the performance of the ASR PBS. We first investigate the effect of etch depth fabrication error, distinguishing between the two types of errors: an underetched grating [see Fig. 4(a), i.e., part of the last thin $\mathrm{Si}$ layer remains on the $\mathrm{SiO}_{2}$ substrate], and an overetched grating [see Fig. 4(b), i.e., the substrate is slightly etched). The results of our simulation show that the reflectivity for TM polarization is more sensitive to the underetching error than is that for TE polarization [see Fig. 4(a)]. The remaining thin layer of $\mathrm{Si}$ will increase the TM reflectivity, since $\mathrm{Si}$ is a dense medium, resulting in a large reduction in the TM transmission efficiency. This also decreases the reflection polarization extinction ratio. 

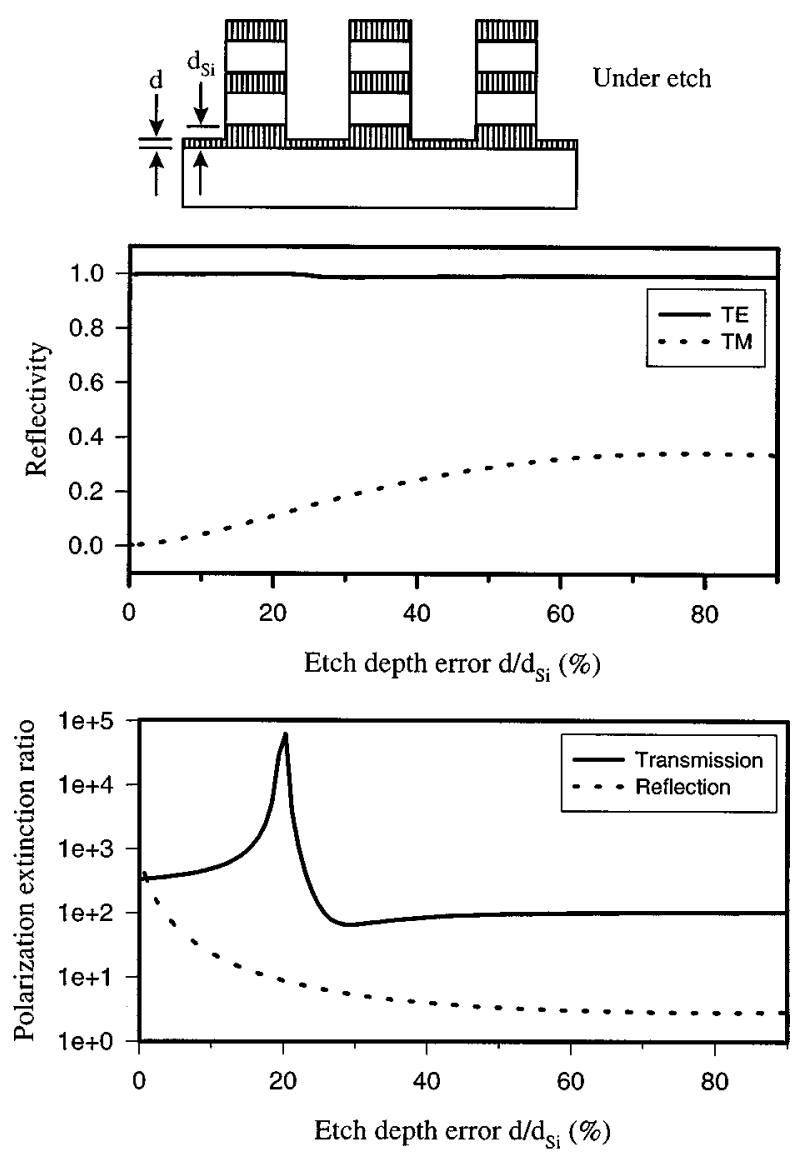

(a)
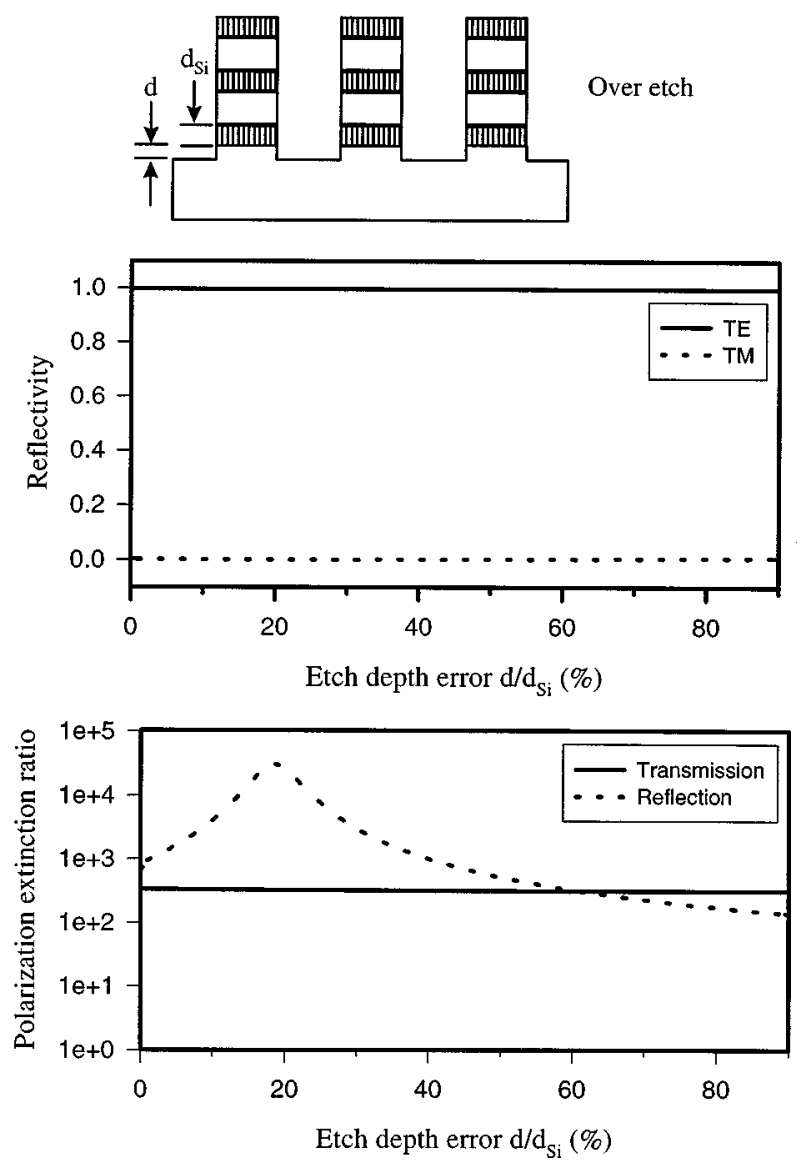

(b)

Fig. 4. Effects of (a) underetching and (b) overetching fabrication error on reflectivity and extinction ratios for TE- and TM-polarized light.

In contrast, Fig. 4(b) shows that overetching error has almost no effect on the efficiency of both TE- and TMpolarized waves. We conclude that an accurate etch depth control to avoid underetching is necessary to attain good performance from these devices.

Another possible fabrication error is the duty ratio error of the high-spatial-frequency grating. We use the same design parameters as for the PBS shown in Fig. 3 (a), except that the duty cycle $F$ is varied continuously from 0.3 to 0.6 [see Fig. 5(a)]. The numerical results indicate that the reflectances for both polarizations stay approximately the same for the different duty cycles ranging from 0.3 to 0.55 [see Fig. 5(b)]. The abrupt decrease of the TE reflectance at the duty cycle of 0.57 may be due to the energy coupling into the guided waves propagating along the multilayer structure. We can observe from Fig. 5 (c) that, when the duty cycle of the grating is approaching 0.54 , the transmission polarization extinction ratios are briefly increasing. Since the polarization extinction ratio is a ratio of a large value to an extremely small value, a minor variation of the small value will cause an abrupt change of the ratio. These resonance phenomena can also be seen in the other figures (see, e.g., Fig. 4).

The last fabrication error that we investigated is the shape of the grating profile. Again, we used the same basic design shape as that of the PBS shown in Fig. 3(a).
We vary the shape of the grating profile by holding the width $W$ of the bottom of the grating constant as the top is varied as $W-\Delta W$ to form a symmetric trapezoidal profile, as illustrated in Fig. 6(a). The results shown in Fig. 6(b) indicate that the efficiencies of the PBS will remain nearly $100 \%$ over a fairly large grating profile error. The polarization extinction ratios [see Fig. 6(c)] slowly decrease as the shape changes from rectangular to trapezoidal.

In general, the ASR PBS are sensitive to underetching fabrication errors. However, these elements are relatively immune to the effects of fabrication errors in duty cycle, grating profile, and overetching. This indicates a rather large fabrication error tolerance of the ASR PBS. In Section 5 we discuss fabrication and experimental verification of such ASR PBS.

\section{FABRICATION AND EXPERIMENTAL CHARACTERIZATION OF THE POLARIZING BEAM SPLITTER}

Fabrication of the ASR PBS in the visible spectral range is a challenging task because of the requirement to fabricate a grating with a subwavelength grating period. However, for near-infrared range of operation, fabrication of the structure is practically possible. For example, in 
our design the total grating depth of five layers is 0.91 $\mu \mathrm{m}$, with a grating period of $0.6 \mu \mathrm{m}$ and a duty cycle of 0.5 , resulting in a grating aspect ratio of approximately $3: 1$, which is within the fabrication capabilities of modern microfabrication technology.

The fabrication procedures of the ASR PBS are shown schematically in Fig. 7. First, five layers of $\mathrm{Si}$ and $\mathrm{SiO}_{2}$, with thickness of 0.13 and $0.26 \mu \mathrm{m}$, respectively, were sputtered alternatively onto a $\mathrm{SiO}_{2}$ substrate to form a one-dimensional (1D) dielectric mirror base. The thickness of each layer was controlled with an accuracy of better than $5 \mathrm{~nm}$. $30-\mathrm{kV}$ high-voltage $e$-beam lithography was then used to define a high-resolution grating with a period of $0.6 \mu \mathrm{m}$ and a duty cycle of 0.5 over a square area of $50 \mu \mathrm{m} \times 50 \mu \mathrm{m}$ on a $0.4-\mu \mathrm{m}$-thick PMMA layer that was thicker than the most conventional $e$-beam lithography resists [see Fig. 7(a)]. The PMMA pattern was developed for $60 \mathrm{~s}$ in a 3:7 mixture of cellusolve and methanol, resulting in a structure shown in Fig. 7(b). Afterward, $0.1 \mu \mathrm{m}$ of chrome was deposited [see Fig. 7(c)],

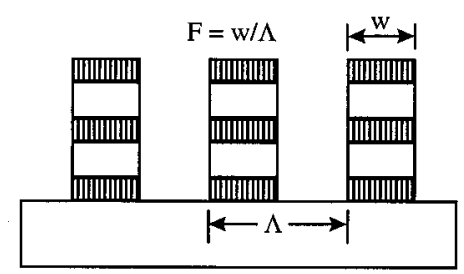

(a)

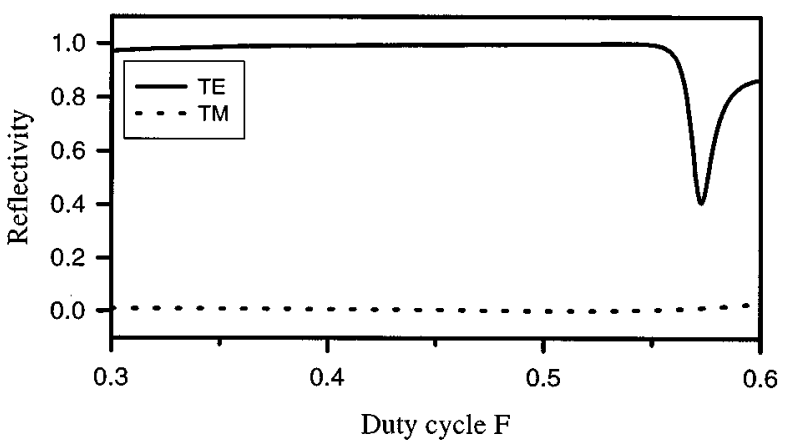

(b)

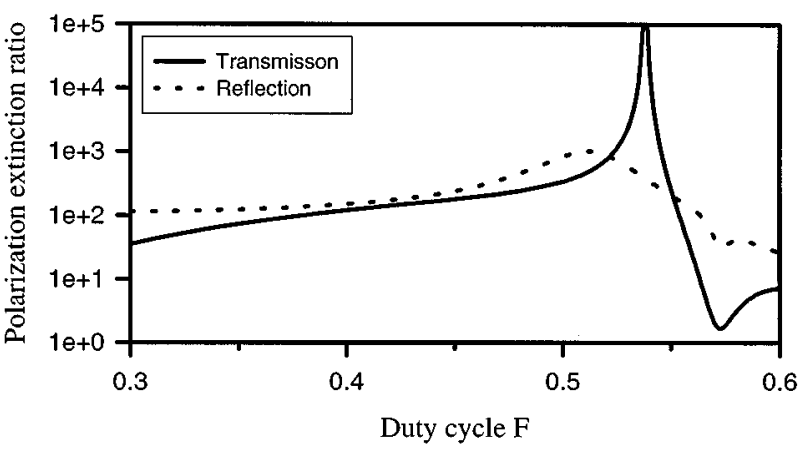

(c)

Fig. 5. (a) Diagram defining duty cycle error. Effect of duty cycle error on (b) reflectivity and (c) extinction ratios of TE- and TM-polarized light.

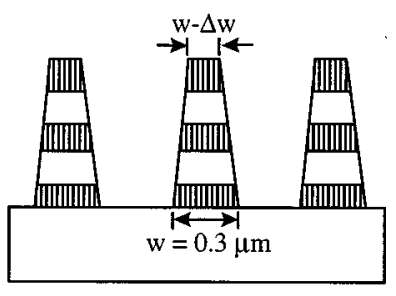

(a)

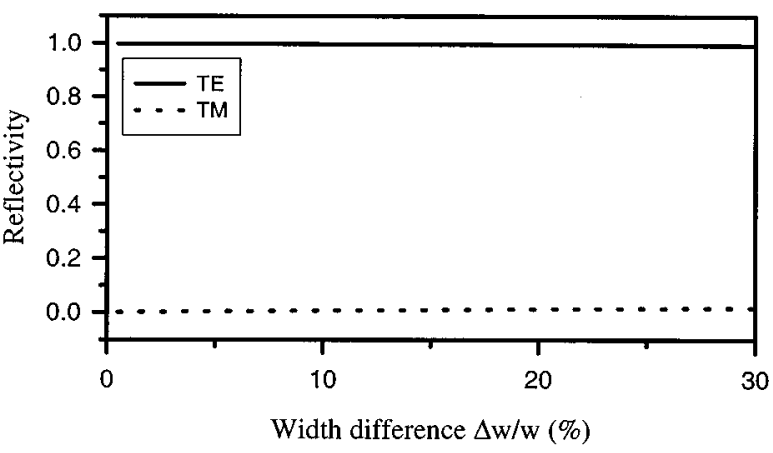

(b)

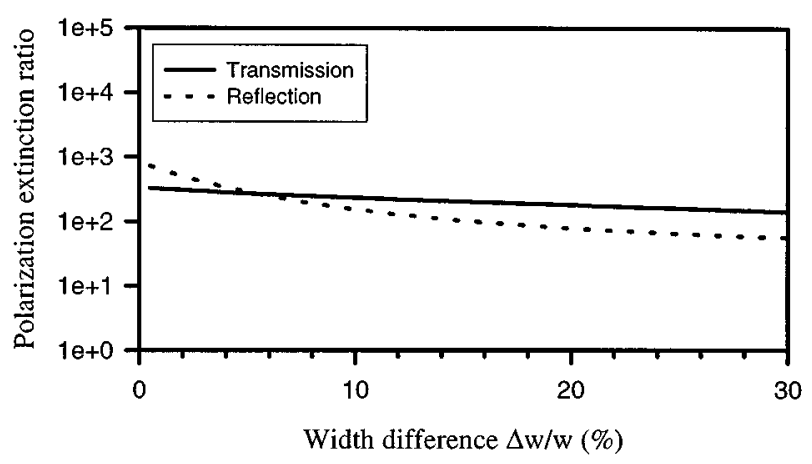

(c)

Fig. 6. (a) Diagram defining grating shape error causing trapezoidal profile. Effect of grating profile error on (b) reflectivity and (c) extinction ratios for TE- and TM-polarized light.

and the PMMA pattern was lifted off in acetone to generate the mask [see Fig. 7(d)]. With the durable chrome mask, the periodic pattern was transferred through the $\mathrm{SiO}_{2}$ and the $\mathrm{Si}$ layers by reactive ion etching with $\mathrm{C}_{2} \mathrm{~F}_{6}$ and $\mathrm{NF}_{3} / \mathrm{CCl}_{2} \mathrm{~F}_{2}$, respectively [see Fig. 7(e)]. The etch rate of $\mathrm{SiO}_{2}$ and $\mathrm{Si}$ was 25 and $100 \mathrm{~nm} / \mathrm{min}$, respectively. Finally, the remained chrome mask was removed by wet etching [see Fig. 7(f)]. The fabricated PBS was inspected under a scanning electron microscope (SEM). The SEM's side view of the structure is shown in Fig. 8.

The fabricated PBS element was evaluated experimentally by means of a measurement setup shown schematically in Fig. 9. We used a polarized $\mathrm{He}-\mathrm{Ne}$ laser source (Melles Griot) operating at a near-infrared wavelength of $1.523 \mu \mathrm{m}$ with a $0.8-\mathrm{mW}$ maximum output power. The laser beam is focused onto the $50 \mu \mathrm{m} \times 50 \mu \mathrm{m}$ aperture of the fabricated element with a low-power $(5 \times)$ microscope objective. The input polarization is controlled by a polarization rotator. Two Ge photodetectors (Newport 


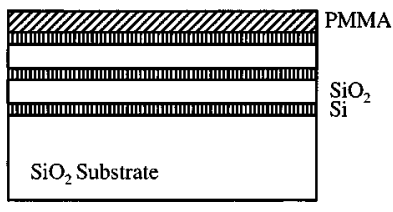

(a) $\mathrm{SiO}_{2}, \mathrm{Si}$, and PMMA deposition

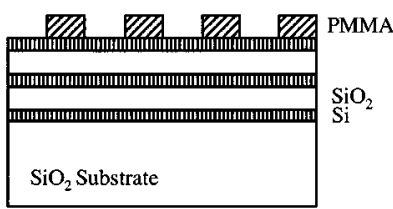

(b) E-beam lithography

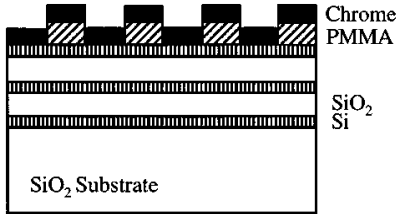

(c) Chrome deposition

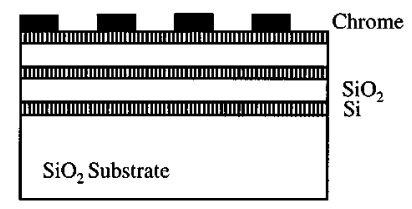

(d) Chrome lift off

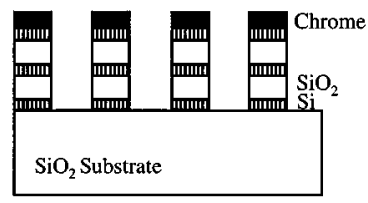

(e) Reactive ion etching

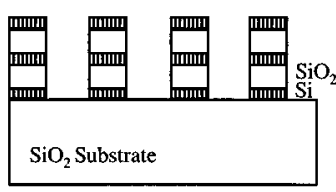

(f) Chrome removal (wet etching)
Fig. 7. Schematic diagram describing the fabrication procedures of ASR PBS on $\mathrm{SiO}_{2}$ substrates. PMMA, poly(methyl methacrylate).

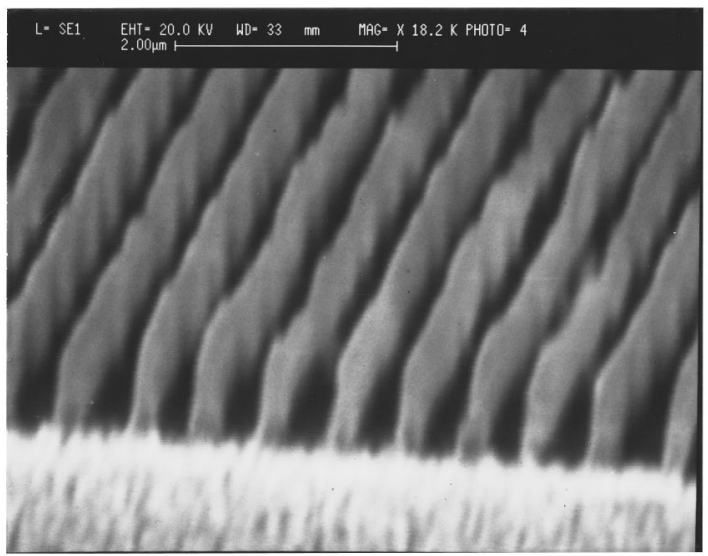

Fig. 8. SEM photograph of the fabricated ASR PBS. The PBS is fabricated on a $\mathrm{SiO}_{2}$ substrate consisting of a multilayer structure of $\mathrm{Si}$ and $\mathrm{SiO}_{2}$ with a thickness of 0.13 and $0.26 \mu \mathrm{m}$, respectively. The grating has a period of $0.6 \mu \mathrm{m}$, with a duty cycle of 0.5 .

Model 818-IR) were used to measure the transmittance and the reflectance simultaneously. For the alignment purpose we also used another $\mathrm{He}-\mathrm{Ne}$ laser operating at a visible wavelength of $0.6328 \mu \mathrm{m}$. The axis of the visible $\mathrm{He}-\mathrm{Ne}$ laser was aligned to coincide with the optical axis of the infrared laser.

The measured efficiencies (i.e., the TM transmittance and the TE reflectance) and the measured polarization extinction ratios versus the incidence angles varying from $32^{\circ}$ to $52^{\circ}$ are shown in Figs. 10(b) and 10(c), respectively. The incidence angle is determined by the angle between the incident beam and the normal to the grating surface. The incident beam is lying in the plane perpendicular to the grating grooves and parallel to the grating vector. In such an arrangement, the measured 1D efficiency and polarization extinction ratio curves correspond to those determined numerically from the vertical cross sections of the $2 \mathrm{D}$ contour plots shown in Fig. 3. The reflectance is measured in a range of $36-52^{\circ}$ because of the practical constraints in the components used in our experimental setup. The experimental results show that the fabricated ASR PBS retains high-polarization extinction ratios over a large angular bandwidth ( \pm 10 degree) from the designed incidence angle of $42^{\circ}$. The measured transmission polarization extinction ratios are higher than 220:1, with a maximum value of 830:1. For the reflection, the polarization extinction ratios are smaller, but they are still better than 40:1, with a maximum value of 70:1. The fabricated ASR PBS also has very high efficiencies. The measured reflection efficiency for the TE-polarized light and transmission efficiency for the TM-polarized light are higher than 99\% and 97\%, respectively. The slightly lower measured efficiency for the TM-polarized light may occur because of the etch depth errors, causing reductions of reflection polarization extinction ratios. We expect that more-accurate control of fabrication tolerances (i.e., etch depth) will improve both the efficiencies and the extinction ratios.

For comparison between the experimental results and the numerical design predictions, we used the SEM image (see Fig. 8) and the optical microscope observation of the fabricated element to estimate the fabrication tolerances of our sample. The sample observed under the optical microscope shows that the $\mathrm{SiO}_{2}$ substrate areas have a light brown color, indicating that the last Si layer was underetched. The SEM photograph indicates a deviation of the grating profile from exact rectangular shape. For modeling purposes we estimate that our sample is $6 \mathrm{~nm}$ underetched. Consequently, we use the modified grating profile shown in Fig. 10(a). Figures 10(b) and 10(c) show that the experimental results and the numerical predictions are in good agreement. Note that the only significant deviation between modeling and experimental results occurs in Fig. 10(c) near the incidence angle of $46^{\circ}$. This deviation may occur because of the limited dynamic range of our photodetectors (i.e., a dynamic range of more than $10^{5}$ will be needed to resolve this resonance).

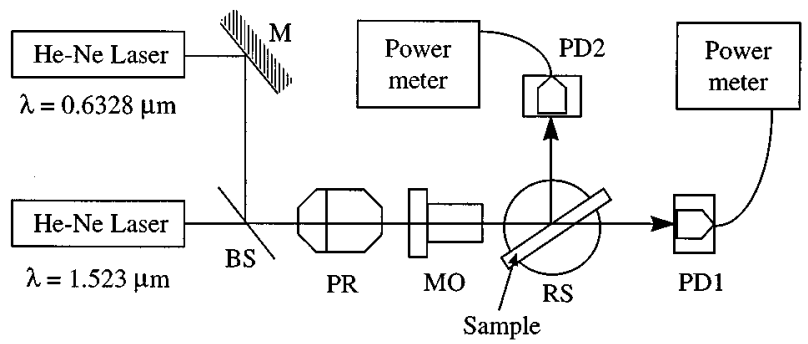

Fig. 9. Schematic diagram of the experimental setup for the characterization of the fabricated ASR PBS. M, mirror; PR, polarization rotator; $\mathrm{BS}$, beam splitter; $\mathrm{MO}$, microscope objective; RS, rotation stage; PD1 and PD2, Ge photodetectors. The transmittance and the reflectance are measured simultaneously to ensure accurate comparison of the extinction ratios and efficiencies. 


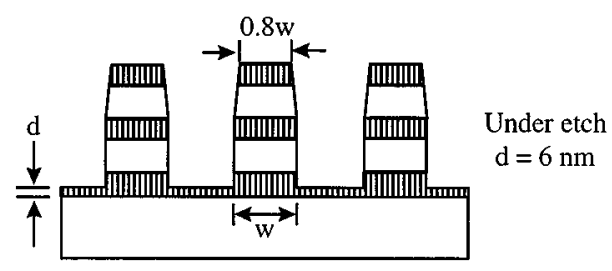

(a)

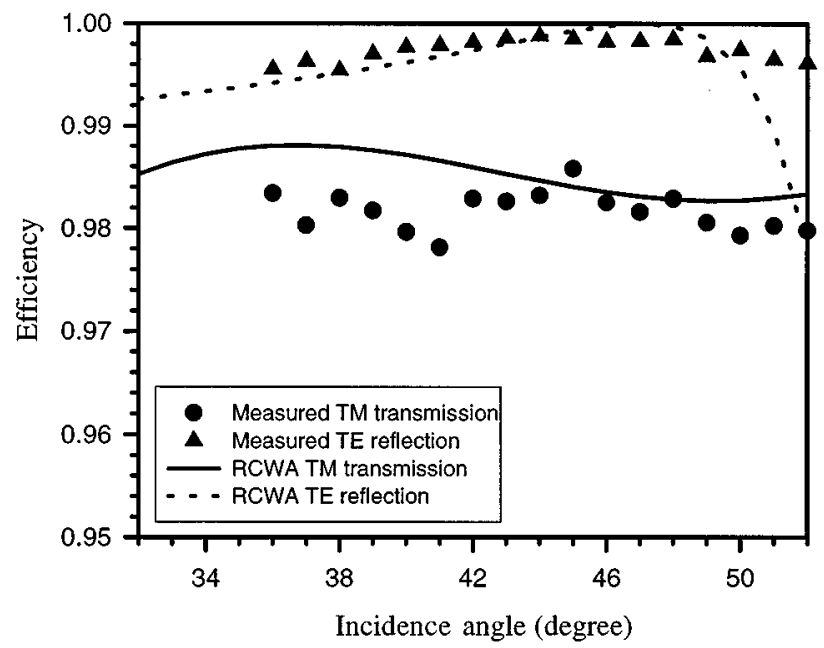

(b)

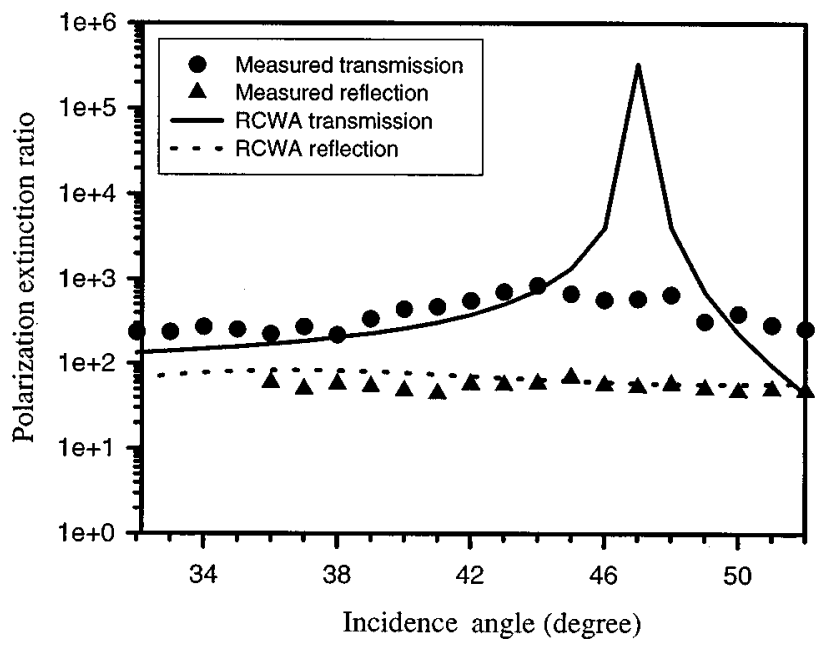

(c)

Fig. 10. Comparison of experimental measurements and numerical predictions of the fabricated ASR PBS. (a) The device structure shown in Fig. 3(a) has been modified to account for the fabricated grating profile error as well as the underetching fabrication error. (b) Measured and calculated efficiencies for TEand TM-polarized waves, (c) measured and calculated polarization extinction ratios in transmission and reflection versus incidence angle.

\section{CONCLUSIONS}

We described the design, fabrication, and experimental evaluations of an ASR PBS. This novel element combines the form-birefringence effect of a high-spatialfrequency grating with the high reflectance of multilayer structures. We use EMT for initial design and RCWA for optimization and numerical characterization of the PBS. Numerical modeling has also shown that these elements can tolerate broad-spectrum optical radiation with good performance in a wide $2 \mathrm{D}$ angular bandwidth range. We have demonstrated that our PBS can be designed to adapt easily to different applications, such as highly efficient polarization-selective mirrors for vertical-cavity microlaser and broadband low-insertion-loss normal-incidence polarizers. Other ASR PBS designs for specified spectrum ranges can be attained by means of suitable combinations of materials to construct the multilayer structures. This design flexibility shows how ASR PBS can address the needs of many different optoelectronic packaging systems. We also discussed the influence of different fabrication errors on the performance of the PBS and observed a rather large fabrication error tolerance of the device.

A sample of the ASR PBS designed for a wavelength of $1.523 \mu \mathrm{m}$ has been fabricated and experimentally characterized. The experimental results show that our PBS provides high measured polarization extinction ratios (maximum value of 830:1) for the two orthogonally polarized output optical waves. The device has also been shown to have the capabilities for operating with optical signals of wide angular bandwidth $\left( \pm 10^{\circ}\right.$ near the designated incidence angle of $42^{\circ}$ ) with a high extinction ratio $(>220: 1)$ and high efficiencies ( $>97 \%)$. Finally, the experimental results have been compared with the numerical predictions and have been found in good agreement. Our future research goals are directed toward enhancing the fabrication precision and toward developing new ASR PBS designs with suitable materials for other spectrum range.

The ASR PBS combines such unique features as compactness, compatibility with semiconductor materials, negligible insertion losses, polarization selectivity for light at normal incidence, high polarization extinction ratios, and operation with waves of large angular bandwidth and from a broad spectral range. These characteristics make the devices desirable for use in image processing, in optical interconnections, and in many other polarization optics applications.

\section{ACKNOWLEDGMENTS}

The authors thank Paul Shames for helpful discussions and preparation of the manuscript. The research conducted at the University of California, San Diego is supported in part by the National Science Foundation, the U.S. Air Force Office of Scientific Research, and the Rome Laboratory. The Research conducted at Caltech is supported by the National Science Foundation.

\section{REFERENCES}

1. F. B. McCormick, F. A. P. Tooley, T. J. Cloonan, J. L. Brubaker, A. L. Lentine, R. L. Morrison, S. J. Hinterlong, M. J. Herron, S. L. Walker, and J. M. Sasian, "Experimental investigation of a free-space optical switching network by using symmetric self-electro-optic-effect devices," Appl. Opt. 31, 5431-5446 (1992).

2. Q. W. Song, M. C. Lee, and P. J. Talbot, "Polarization sensitivity of birefringent photorefractive holograms and its 
applications to binary switching," Appl. Opt. 31, 6240-6246 (1992).

3. Y.-T. Huang and Y.-H. Chen, "Polarization-selective elements with a substrate-mode grating pair structure," Opt. Lett. 18, 921-923 (1993).

4. M. Ojima, A. Saito, T. Kaku, M. Ito, Y. Tsunoda, S. Takayama, and Y. Sugita, "Compact magnetooptical disk for coded data storage," Appl. Opt. 25, 483-489 (1986).

5. R. K. Kostuk, T.-J. Kim, G. Campbell, and C. W. Han, "Diffractive-optic polarization-sensing element for magneto-optic storage heads," Opt. Lett. 19, 1257-1259 (1994).

6. P. Kunstmann and H.-J. Spitschan, "General complex amplitude addition in a polarization interferometer in the detection of pattern differences," Opt. Commun. 4, 166-168 (1971).

7. L. B. Wolff, "Polarization camera for computer vision with a beam splitter," J. Opt. Soc. Am. A 11, 2935-2945 (1994).

8. K. Shiraishi, T. Sato, and S. Kawakami, "Experimental verification of a form-birefringent polarization splitter," Appl. Phys. Lett. 58, 211-212 (1991).

9. J. L. Pezzaniti and R. A. Chipman, "Angular dependence of polarizing beam-splitter cubes,” Appl. Opt. 33, 1916-1929 (1994).

10. S. Aoyama and T. Yamashita, "Grating beam splitter polarizer using multi-layer resist method," in International Conference on the Application and Theory of Periodic Structures, J. M. Lerner and W. R. McKinney, eds., Proc. SPIE 1545, 241-250 (1994).

11. M. C. Gupta and S. T. Peng, "Multifunction grating for signal detection of optical disk," in Optical Data Storage '91, T. A. Shull, N. Imamura, and J. J. Burke, eds., Proc. SPIE 1499, 303-306 (1991).

12. S. Habraken, O. Michaux, Y. Renotte, and Y. Lion, "Polarizing holographic beam splitter on a photoresist," Opt. Lett. 20, 2348-2350 (1995)

13. R. M. A. Azzam, "Polarizing beam splitters for infrared and millimeter waves using single-layer coated dielectric slab or unbacked films," Appl. Opt. 25, 4225-4227 (1986).

14. R. C. Tyan, P. C. Sun, A. Scherer, and Y. Fainman, "Polarizing beam splitter based on anisotropic spectral reflectivity characteristics of form-birefringent multilayer gratings," Opt. Lett. 21, 761-763 (1996).

15. S. M. Rytov, "Electromagnetic properties of a finely stratified medium," Sov. Phys. JETP 2, 466-475 (1956)

16. M. G. Moharam and T. K. Gaylord, "Diffraction analysis of dielectric surface-relief gratings," J. Opt. Soc. Am. 72, 1385-1392 (1982).

17. I. Ricther, P. C. Sun, F. Xu, and Y. Fainman, "Design considerations of form birefringent microstructures," Appl. Opt. 34, 2421-2429 (1995).

18. D. F. Edwards and H. R. Philipp, in Handbook of Optical Constants of Solids, E. D. Palik, ed. (Academic, Orlando, Fla., 1985), pp. 547 and 749

19. E. Hecht, Optics (Addison-Wesley, Reading, Mass., 1990), p. 377.

20. E. D. Palik, in Handbook of Optical Constants of Solids, E. D. Palik, ed., (Academic, Orlando, Fla., 1985), p. 695.

21. F. Xu, R.-C. Tyan, P.-C. Sun, Y. Fainman, C.-C. Cheng, and A. Scherer, "Fabrication, modeling, and characterization of form-birefringent nanostructures," Opt. Lett. 20, 24572459 (1995).

22. M. Born and E. Wolf, Principles of Optics (Pergamon, Oxford, 1975), pp. 705-708.

23. Reference 22, pp. 51-70.

24. C. W. Haggans, L. Li, and R. K. Kostuk, "Effective-medium theory of zeroth-order lamellar gratings in conical mountings,” J. Opt. Soc. Am. A 10, 2217-2225 (1993).

25. H. Kikuta, H. Yoshida, and K. Iwata, "Ability and limitation of effective medium theory for subwavelength gratings," Opt. Rev. 2, 92-99 (1995).

26. F. Xu, R.-C. Tyan, Y. Fainman, and J. E. Ford, "Singlesubstrate birefringent computer-generated holograms," Opt. Lett. 21, 516-518 (1996). 